\title{
El control de gestión en el sector público: su aplicación en el MOPTMA
}

\section{José Carlos Alcalde Hernández*}

\section{El control interno: fundamento}

- Existe unanimidad en considerar que el control es una necesidad inherente al proceso de "complicación" orgánica que supone abandonar fórmulas cuasi-individuales de actuación. Surgiría el control precisamente cuando el responsable (dueño) de la organización no puede asumir personalmente las tareas de vigilancia y supervisión que toda dirección implica y, en otro sentido, cuando las lineas operativas se separan de la dirección hasta el punto de recibir las órdenes no directamente sino a través de "intermediarios".

En este punto inicial conviene hacer una primera delimitación: entendemos que existe control cuando se "institucionalizan" de algún modo en un núcleo de la organización una serie de tareas específicas de vigilancia y revisión, con independencia o al margen de las que naturalmente implica una genérica actividad directiva. Ello nos lleva al mismo tiempo a diferenciar el "control interno", del que en adelante vamos a tratar, de los denominados "sistemas o mecanismos de control interno" en la práctica auditora, con los que se alude a trámites o elementos de los procedimientos, que no tienen por qué estar institucionalizados en un órgano especial y cuya finalidad es introducir garantías o seguridad en el funcionamiento (revisión de lo actuado por un superior; segregación de funciones, etc.).

El fenómeno de diversificación organizativa no se limita, lógicamente, a un mero alejamiento, sino que incorpora otros factores de complejidad en el funcionamiento (desde el mismo proceso de toma de decisiones, con la participación integrada de diversas áreas, la planificación, etc.); esta complejidad refuerza la necesidad del control, no sólo esta vez como mecanismo de comprobación del cumplimiento de las decisiones, sino como fuente de información del grado de ajuste a las mismas de la organización, a efectos de su adaptación o modificación, etc., (realimentación).

Desde otra perspectiva pero siempre con esta nota de instrumento de dirección, se ha destacado el papel de la actividad de control en orden a la "estandarización" en el funcionamiento de las diversas áreas de la organización, como medio sustitutorio de la supervisión directa y continuada. Superada la simplicidad orga- nizativa, la unión o coordinación de funcionamiento de las diversas áreas suele basarse en la homogeneización de procedimientos y/o de productos, que requiere la fijación previa de pautas o reglas de actuación sobre cuyo seguimiento, para resultar eficaz, debe tenerse un elevado grado de seguridad.

- La verificación del ajuste a las directivas, en tanto objeto básico del control no puede convertirse en su propia finalidad (sería tanto como "controlar por controlar"). Esa verificación se efectúa para comunicar sus resultados al "interesado" en el control, que en el caso del control interno como vimos es "la dirección". La dirección empleará los resultados del control para adoptar sus decisiones (confirmar orientaciones, reorientar, "sancionar" desviaciones ....). Por otro lado, el control al detectar y comunicar posibles incorrecciones (en la mayoría de los casos "no maliciosas" sino debidas a defectos procedimentales), está posibilitando su rectificación y no reproducción.

- Complementariamente el control interno cumple una función de garantia o confianza, también de gran importancia en el funcionamiento de una organización, tanto para la dirección sobre el seguimiento de sus instrucciones, como incluso para terceros, interesados en relación con la existencia de mecanismos internos dirigidos a velar por una actuación adecuada.

- En definitiva, el control interno es un instrumento de la organización para lograr una gestión cualitativa (y cuantitativamente) mejor y la "mejora de la gestión" es la finalidad básica del control interno

Diferencia fundamental con el control externo cuya finalidad es "comunicar" valoraciones de la gestión a terceros con interés efectivo en su resultado pero ajenos propiamente a la organización controlada, y que, con independencia de su preocupación por la buena marcha de los asuntos, normalmente utilizarán el control para adoptar decisiones relativas a sus intereses específicos.

- Las características específicas y delimitadoras de la función de control interno se relacionan con el alcance que demos a dichos términos:

a) En cuanto control, la actuación es de revisión o verificación de lo que hacen o realizan otros elementos de la organización. 
b) También en cuanto control, la actuación no se dirige directamente a producir un bien o servicio propio del objeto o de la actividad que justifica la existencia del ente, sino a efectuar un examen, precisamente, por lo general, de dicha estructura operativa y de su organización.

c) La actuación de control tiene como efecto "natural" una manifestación o traslado de sus resultados a otros órganos, que se servirán de ellos, para adoptar decisiones. Los resultados del control revertirán, indudablemente, en la experiencia y proyección de esta actividad, pero su destino directo no es la utilización por el propio control.

d) La actividad del "controlador" es fundamental y primordialmente "crítica", con independencia de que la misma se oriente en un sentido constructivo. Ello permite diferenciar esta actuación de otras en las que igualmente se produce una aportación de opinión al órgano decisorio (asesoría, informe), pero con una finalidad directa de aportar datos para facilitar su decisión (de ilustrar).

No obstante, es frecuente que el órgano de control, dada su especialización, sea utilizado en numerosas ocasiones como órgano asesor. Pero en todo momento convendrá delimitar cuándo se actúa en uno u otro sentido, pues de lo contrario se corre el riesgo de una desnaturalización de la función de control, riesgo más acentuado cuando el asesoramiento se realice al mismo órgano controlado.

e) Una consecuencia de esa actitud critica, y en cierto modo del distanciamiento y objetividad de la actuación de control, es que su destinatario directo no es sólo el propio órgano controlado, pues en todo caso debe existir la posibilidad de traslado de sus conclusiones a otra instancia distinta y superior.

f) El control implica, además, la ausencia de facultades decisorias sobre el comportamiento controlado; tanto en lo que se refiere a los actos operativos a efectuar como a la adopción de medidas correctoras de los ya realizados.

Se ha dicho en este sentido que el control representa una declaración de "juicio" frente a la declaración de "voluntad" que comporta una decisión, y ello ha de ser así, incluso en los casos (normalmente de control previo) en los que al control se reconozcan efectos suspensivos o paralizantes de las propuestas examinadas: tampoco en estos supuestos el control decidirá, y de ahí la necesidad de que el conflicto sea resuelto por un órgano superior diferenciado, también desde luego del controlado.

g) Dado su carácter de "interno", el control sirve exclusivamente a la organización, esto es, sus resultados quedan dentro de ésta sin trascendencia exterior; sólo el cumplimiento de específicas reglas imperativas puede determinar una salida al exterior de dichos resultados. h) Pero el carácter de interno no disminuye una nota ineludible en toda actuación de control: la independencia y autonomia respecto del controlado, mecanismo además de garantía de la finalidad que persigue el control.

La independencia necesita asegurarse con una serie de notas adjetivas, entre las que la profesionalidad y competencia del personal encargado de su realización son de especial trascendencia.

i) El carácter interno implicará la dependencia orgánica de una área de la propia organización, siempre al nivel preciso para asegurar la autonomía respecto de las zonas controladas.

El órgano de dependencia deberá ser el receptor primario de los resultados del control y el que puede adoptar tanto decisiones consecuencia de los mismos de cara a los controlados como instrucciones en cuanto a la realización de los trabajos de control; pero también respecto de este órgano de dependencia el controlador debe sentirse profesionalmente "independiente" en cuanto a las valoraciones a incluir en sus informes y al mismo tiempo admitir que la adopción de medidas posteriores corresponde al órgano directivo y no al controlador.

\section{Control en el sector público}

Hecha esta primera aproximación al fenómeno del control interno, podemos ahora dirigirnos hacia el otro elemento de referencia de este artículo: la gestión pública; es decir, el carácter público del sujeto y de la actividad controlados a fin de determinar las posibles especialidades que de ello pueden derivarse.

En primer lugar y desde un punto de vista subjetivo no parece necesario insistir en destacar la complejidad del sector público, tanto organizativa (número de agentes, diferente tipología, estructuras internas con gran diversidad de instancias) como material (actividades económicas, sociales, de carácter general y burocrático), así como el importante volumen de recursos que gestiona. De todos modos, estas características distintivas habrán de ser consideradas a la hora de comprender adecuadamente el sentido y alcance del control a desarrollar.

Nos detendremos a nuestros efectos en dos aspectos fundamentalmente: la estructuración básica del sector público (estatal) y las peculiaridades generales más notables de la gestión pública.

\section{II.1 Modelo general de organización}

Quizás para algunos resulte cuestionable la existencia misma de un "modelo" organizativo teniendo en cuenta la situación actual, fruto del proceso de reforma seguido en los últimos años. 
En principio, las medidas adoptadas se han orientado buscando fórmulas organizativas que posibilitaran, en un sentido genérico, formas de actuación gerenciales, bien dentro de los tipos "clási-

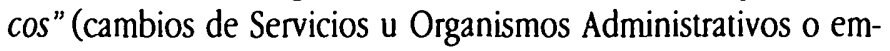
presariales) o bien haciendo aparecer un conglomerado nuevo y "atípico" con estarutos particulares (el fenómeno de los Entes Públicos y Agencias).

La configuración de este marco es, pues, el resultado de un proceso, por otro lado no exclusivo de nuestros dias, impulsado por una idea central: búsqueda de mecanismos de gestión más flexibles que los estrictamente administrativos y más adecuados con las exigencias de los servicios a prestar.

Si algo caracteriza al vigente modelo organizativo público es la ausencia de un marco general y estable; de todos modos, a efectos sistemáticos podríamos elaborar el siguiente esquema:

\begin{tabular}{|l|l|}
\hline $\begin{array}{l}\text { Sector puramente } \\
\text { administrativo }\end{array}$ & $\begin{array}{l}\text { Ministerios } \\
\text { O.A. Administrativos }\end{array}$ \\
\hline $\begin{array}{l}\text { Sector intermedio } \\
\text { (t-administrativo) }\end{array}$ & $\begin{array}{l}\text { O.A. CIF } \\
\text { Entes Públicos (Agencias) }\end{array}$ \\
\hline Sector empresarial & $\begin{array}{l}\text { Entes Públicos } \\
\text { Sociedades estatales mercantiles }\end{array}$ \\
\hline
\end{tabular}

El sector puramente administrativo se caracterizaría por la existencia de un rigido marco de actuación, impuesto externamente, donde la capacidad de maniobra del gestor se encuentra circunscrita al cumplimiento de normas procedimentales y todo ello como consecuencia de las exigencias del principio de legalidad en general y de legalidad financiera en particular. En principio ello va unido a una prevalencia del procedimiento (¿cómo se hacen las cosas?) sobre los resultados (¿cuántas se hacen?).

Desde el punto de vista presupuestario rige el Presupuesto limitativo y vinculante para la gestión.

El sector empresarial recogería en mayor medida la "autonomía" del propio gestor en el contenido y configuración de sus relaciones, consecuencia de la importancia que adquiere la obtención de resultados y objetivos económicos. El Presupuesto tiene un carácter estimativo y flexible, acorde con la naturaleza económica de las acciones desarrolladas. En la situación actual se englobarían bajo esta rúbrica entes que actúan para prestar servicios económicos, no todos en régimen de competencia.

Pero ambos sectores son "públicos". En principio, ello implica que en gran parte se financian con recursos públicos (vía impositiva); si bien este dato habría de completarse con la idea más elemental de que los resultados de la gestión en última instancia repercutirán en las cuentas públicas (positiva o negativamente).

Una característica a considerar es que en el sector público existe una estrecha conexión entre tipo de órgano/sistema de gestión. El diseño organizativo no parte tan sólo de una búsqueda para hacer las cosas "más organizadamente", (desde un punto de vista subjetivo o de unidad actora) sino de un deseo de poder hacer las cosas de otra manera (en un sentido de gestión o procedimental). En definitiva, las posibilidades organizativas existentes no sólo ofrecen distintas vías de distribución de las funciones sino sistemas diferentes de gestión.

En este sentido, a la hora de decidir la configuración de muchos supuestos, problemas esenciales de análisis orgánico (como distribución de competencias; relaciones entre áreas de actuación; configuración de mecanismos de control de gestión), han sido desplazados por cuestiones más típicamente funcionales como regulación del status jurídico del personal; Derecho aplicable en las relaciones externas; régimen jurídico presupuestario y patrimonial 0 modificación de controles administrativos.

Finalmente, resaltar que el modelo está construido sobre la particularización, en todo aquello que implique diferencia respecto del núcleo básico Estado/Organismos Autónomos; particularización a través del Estatuto de cada Ente que engloba diferentes alternativas de gestión, presupuestación, contabilización y control.

Desde este punto de vista del sujeto controlado, cabría apuntar que el control, aunque encuentre una referencia orgánica común, como medio de potenciar su autonomía, debe adaptarse a los diferentes tipos y modos de gestión, de forma que cada uno de ellos se controle mediante los procedimientos y métodos adecuados a la naturaleza y requerimientos de la actividad controlada.

\section{II.2 Exigencias de la Gestión Pública}

A los efectos de nuestro análisis se trataría de contemplar la gestión pública desde la óptica de una serie de criterios de racionalidad elemental, que deben inspirar su desarrollo y que de algún modo la caracterizan frente a la gestión privada, pero no hasta el punto de que podamos considerar ambos sectores aislados uno del otro; al contrario, seguramente la zona de confluencia es mayor que la exclusiva, y tiende a aumentar si la comparación se hace con las organizaciones privadas de mayor complejidad.

1) En primer lugar, pueden considerarse tres notas esenciales de la gestión pública como gestión de intereses ajenos, de intereses públicos y de fondos públicos.

Gestión de intereses ajenos, en cuanto que propiamente los asuntos que se gestionan no son de interés directo y particular de los propios gestores, que actúan, en cierto modo, como mandatarios de los titulares reales de dichos intereses; la visión de la Administración Pública como mandataria de los ciudadanos se encuentra en la esencia misma del Estado de Derecho.

Gestión de intereses públicos, en el sentido de que los intereses gestionados no pueden asociarse directa y exclusivamente a una persona $\mathrm{o}$ a un colectivo específico y con- 
creto de personas sino que, de alguna manera, se relacionan con los intereses de la colectividad. No se trata aquí de plantear una explicación de la teoría de los intereses públicos, pero en cualquier caso esta idea básica de que se trata de intereses de la colectividad y de que los resultados de la gestión efectuada en relación con dichos intereses van a afectar también a dicha colectividad, son aspectos fundamentales que creo interesante retener. La prevalencia de los intereses públicos frente a los particulares justifica la mayoría de los clásicas "prerrogativas" de la gestión pública.

En tercer lugar, la gestión pública es fundamentalmente gestión de fondos públicos, que a su vez tienen una característica esencial: se obtienen de una manera imperativa e incluso coactiva, al existir la posibilidad de poner en marcha mecanismos ejecutivos de recaudación de lo debido a la colectividad.

Por otro lado, también ha sido una regla general de los Estados modernos, desde el punto de vista financiero, la de que los fondos públicos obtenidos tienen una afectación general al conjunto de los intereses públicos y no particularizada hacia determinados gastos públicos específicos, salvo que como excepción se permita legalmente.

Como consecuencia de ello se establece una organización concreta para recaudar los fondos públicos y, al mismo tiempo, para proponer y materialmente facilitar su distribución centralizada a través del Presupuesto que, a este respecto, comporta una decisión política sobre la distribución de esos recursos generales.

Finalmente conviene destacar, más en los últimos años, que estos recursos han de caracterizarse como recursos escasos; en este sentido, la restricción financiera es un elemento fundamental a la hora de conocer las posibilidades mismas de la gestión pública.

Todos estos aspectos han de ser apreciados a la hora de caracterizar la gestión pública, sobre todo si se tiene en cuenta que en la práctica han comportado la existencia de toda una serie de instancias horizontales (de influencia en toda la organización) que a través de autorizaciones y aprobaciones externas condicionan las posibilidades reales del gestor directo.

2) A continuación vamos a plantearnos cuáles son los vectores o líneas de comportamiento fundamentales que enmarcan esta gestión pública y que podemos asociar con tres criterios: un criterio estructural, un criterio operativo y un criterio rector de la toma de decisiones.

Desde un punto de vista estructural o político es de destacar el principio de legalidad. La Administración ha de actuar sometida al conjunto de normas que componen el Derecho y además con una característica esencial en el ámbito del sector más administrativo: se trata de normas impuestas externamente desde el Parlamento o incluso desde el Gobierno que también presenta un cierto carácter extemo respecto de cada gestor concreto.

En segundo lugar, desde el punto de vista operativo también la gestión pública está obligada, y no en menor medida que el sector privado, a desarrollarse con eficacia y eficiencia tanto en cada operación aislada como en el conjunto de la actividad.

Se trata de actuar con eficacia y eficiencia con la misma intensidad que en el sector privado; quizás en este punto lo que no resulte aplicable sean otras guías de actuación como la rentabilidad o la maximización del beneficio al fin de ejercicio, por lo que la eficacia y eficiencia públicas han de ligarse sobre todo a exigencias de actuación racional (cumplimiento de objetivos) y optimización de recursos escasos.

Eficacia y eficiencia que, conviene recordar, no pueden ser predicables exclusivamente como guía de las decisiones del gestor directo, sino que comportan a su vez una serie de medidas organizativas generales que todo gestor público que propugne dichos criterios ha de aceptar y promover: planificación, seguimiento y control de gestión, información a la dirección, análisis de costes, rendición de cuentas, etc

Un tercer criterio, directamente relacionado con la forma de orientar los procedimientos decisorios, sería el de la objetividad que en esencia va a implicar que las decisiones del gestor no van a depender de su exclusiva visión e interpretación "subjetiva".

La objetividad, que conecta directamente con el carácter público de los intereses gestionados y de los fondos que se administran, no es un principio puramente teórico sino que, con independencia de que debe enmarcar toda la normativa rectora de la gestión, comporta una serie de exigencias:

a. Publicidad y concurrencia en todos los procesos de toma de decisiones que afecten a una pluralidad de ciudadanos, cuando son varios los que posiblemente podrían participar de sus consecuencias.

b. En segundo lugar, conocimiento transparente de las peculiaridades del proceso decisorio y de todas aquellas aclaraciones que cualquiera haya podido merecer durante el mismo.

Desde el punto de vista de los ciudadanos, Michel GeNTOR considera que el derecho a la trasparencia de la gestión pública, comprendería:

- el derecho a conocer (acceso, en general, a las informaciones deducidas de las actuaciones administrativas). 
- el derecho a comprender (claridad de los documentos, motivación de decisiones, fiabilidad y publicidad de informes).

- el derecho a ser escuchado (procedimientos contradictorios, trámite de audiencia, planteamiento a información pública de decisiones generales).

- el derecho a criticar (posibilidad de recurrir, en vía jurisdiccional).

c. En tercer lugar, observabilidad de las actuaciones y decisiones tanto por los superiores como por los propios ciudadanos que estén interesados a efectos, por ejemplo, de posibles recursos a interponer y por el distinto conjunto de supervisores. Ello implica exteriorización y concreción de criterios e identificación de quien los asume, así como documentación de actuaciones.

d. Finalmente, aplicación realmente con carácter excepcional de todas aquellas autorizaciones que de algún modo se separan de los criterios generales; autorizaciones que por otro lado igualmente deberán estar motivadas.

Ahora bien, la objetividad en la gestión debe estar a su vez basada en otro aspecto, fundamental por otro lado en la figura misma del gestor de asuntos ajenos: la confianza en el mandatario; la "buena gestión" pública no puede desarrollarse en estado de excepción permanente, o bajo un régimen de continua sospecha o desconfianza respecto del gestor.

Los tres criterios indicados (legalidad, eficacia/eficiencia y objetividad) confluyen en la gestión pública y determinan su alcance; no quiero excluir con ello la existencia de otros criterios rectores del comportamiento público (la austeridad, por ejemplo, me parece un principio que debería considerarse consustancial), pero quizás sean los que en mayor medida configuran el marco y posibilidades reales de los gestores y, en definitiva, las pautas básicas a valorar por el control.

Lo que sí parece importante, como consecuencia además de una cierta experiencia de la realidad cercana, es evitar toda visión "conflictiva" o de preponderancia entre las tres pautas de comportamientu; su ordenación lógica debe llevar en primer lugar a aplicar el cumplimiento normativo, pero considerando que la norma ha de elaborarse desde su dirección a la eficacia y a la objetividad (también evidentemente al respeto de garantías ciudadanas), y revisarse en el sentido de proponer todas aquellas modificaciones que requieran estos dos últimos principios, que a su vez han de ser contrastados permanentemente con la realidad material de los procedimientos de gestión, mucho más compleja que el simple cumplimiento normativo.
En definitiva, pueden existir normas contradictorias con la eficacia y/o la objetividad y normas "perfectas" desde estos puntos de vista pero cuya aplicación práctica y procedimental se aleja de dichos criterios. El gran problema, y quizás también el mayor reto, sería pues el equilibrio y la armonización permanente de los tres principios, y en esta tarea el control interno en este sector puede y debe representar una gran utilidad.

Si bien las notas anteriores son de plena aplicación al que antes denominamos "sector administrativo", no resulta posible excluir su aplicación absoluta en el "sector empresarial", dado su carácter público, y teniendo en cuenta un elemento que por circunstancias conocidas, y lamentables, se ha manifestado con gran fuerza en los últimos tiempos: el factor de exigencia social de rigor y objetividad en el comportamiento de los gestores públicos con independencia de la naturaleza de la actividad desarrollada.

\section{Control interno y control de gestión}

Desde la perspectiva anterior, son numerosas las normas que disciplinan y regulan la gestión de los diferentes agentes públicos, pero junto a ello, ha sido general el establecimiento de instituciones o mecanismos dirigidos precisamente al "control" de dicha gestión, a fin de asegurar en mayor medida su regularidad y sujeción a los principios informadores de lo que podría denominarse "una buena gestión pública".

$\mathrm{Al}$ igual que en numerosos países de la Europa continental, en España tradicionalmente el control de la actuación económica de los agentes públicos se ha orientado en dos sentidos, representados cada uno de ellos por dos instituciones u órganos:

1. El denominado "control externo", ejercido por el Tribunal de Cuentas, directamente dependiente de los Cortes Generales.

2. El "control interno" desarrollado por un órgano, la Intervención General de la Administración del Estado, incluido en la estructura del propio Poder Ejecutivo, concretamente, dentro del Ministerio de Economía y Hacienda.

Conviene apuntar ya desde ahora que este esquema dual se encuentra desde mediados de los años 80 sometido a revisión, no tanto por necesidad de alterar sustancialmente la existencia y funciones de las dos instituciones controladoras, como por completarlo a fin de cubrir adecuadamente las potencialidades del control de cara a la autonomía y responsabilización de los gestores públicos; ello ha conducido a la aparición de un tercer supuesto (controles internos departamentales) que será el núcleo de este artículo.

No obstante, repasaremos brevemente las características esenciales del Tribunal de Cuentas e Intervención General de la Admi- 
nistración del Estado, para ubicar de la manera más adecuada el control que nos interesa.

Realmente el objeto del control ejercido por una y otra institución es idéntico: la actividad de carácter económico-financiero de los Entes de naturaleza pública y, más concretamente, la valoración de la misma en cuanto a su ajuste a la doble exigencia legal (en sentido amplio) y económica (principios de eficiencia y economía), antes señalada. Desde este premisa, existen no obstante una serie de diferencias que, siquiera sea esquemáticamente, conviene indicar:

\begin{tabular}{|l|l|}
\hline \multicolumn{1}{|c|}{ TRIBUNAL DE CUENTAS } & \multicolumn{1}{|c|}{$\begin{array}{c}\text { INTERVENCIÓN GENERAL } \\
\text { DE LA ADMINISTRACIÓN } \\
\text { DEL ESTADO }\end{array}$} \\
\hline $\begin{array}{l}\text { - Control externo: dependiente de las } \\
\text { Contes Generales. }\end{array}$ & $\begin{array}{l}\text { Control interno: órgano del Poder Eje- } \\
\text { cutivo. } \\
\text { - Control siempre ex post. }\end{array}$ \\
$\begin{array}{l}\text { - Control "previo" (función interventora) } \\
\text { yobre todo el sector público (incluidas } \\
\text { Comunidades Autónomas y Corpora- (Control financiero). } \\
\text { ciones Locales). }\end{array}$ & $\begin{array}{l}\text { Sólo el sector público estatal (Admi- } \\
\text { nistración del Estado, Organismos, Em- } \\
\text { presas y Entes dependientes). }\end{array}$ \\
\hline
\end{tabular}

La Intervención General de la Administración del Estado (IGAE) se configura como un control interno, en cuanto se incardina en la propia organización del Poder Ejecutivo. En este sentido, desamolla sus funciones dentro del marco básico al que antes nos referíamos.

No obstante, la IGAE presenta una serie de características que conviene retener:

a) Se trata de un control "General", que se desarrolla respecto de todo el sector público estatal, si bien adecuando su forma de control a las características de sus diferentes agentes.

b) Es un control "horizontal" en cuanto sirve fundamentalmente, dentro del Poder Ejecutivo, a las funciones de un órgano de tal carácter, el Ministerio de Economía y Hacienda, con responsabilidades genéricas en la gestión económica pública, de algún modo superpuestas a las particulares de cada zona de actuación (y que se patentizan en momentos de restricción presupuestaria como los actuales).

Estos dos aspectos han venido presentando el control de la IGAE, desde su inicial afirmación como control interno general, con un carácter de "externo" por lo que respecta a la visión que del mismo tienen los diferentes sectores funcionales objeto del control (por no hablar más concretamente de cada órgano controlado).

Este alejamiento ha sido puesto de relieve en mayor medida en un contexto general tendente a incrementar la autonomía, flexibilidad y responsabilización de los gestores en su procesos estratégicos y operativos.
Es en este contexto donde debemos situar la aparición de los primeros supuestos de control de gestión, utilizando este termino ya desde ahora para referirnos al control interno, no general u horizontal, sino dependiente funcional y orgánicamente del núcleo de la organización efectivamente responsable del desarrollo y efectividad de una determinada y sustantiva zona de actuación pública.

En un sentido más descriptivo podemos decir que el control de gestión es el control "al servicio del gestor" directo.

Junto a ello debe también desde el principio remarcarse que el control de gestión así entendido, en absoluto implica o debe implicar un detrimento en la esencia misma de la función (el control), de forma que en ningún caso debe dudarse de la independencia, profesionalidad y capacidad de los que lo desarrollan y de la fiabilidad de la metodología empleada.

Como antecedentes más inmediatos, las Inspecciones Generales de Servicios (aunque históricamente limitaban sus actuaciones a actividades muy puntuales), vinieron desarrollando las facultades de control que la Ley de Régimen Jurídico de la Administración del Estado de 1957, atribuía a los Subsecretarios de los Ministerios (art. 15).

Estas unidades se han venido configurando como Subdirecciones Generales de las Subsecretarias, con un cierto lazo de unión funcional (no jerárquico) con la Inspección General de Servicios de la Administración Pública (Ministerio para las Administraciones Públicas); existía alguna excepción a este supuesto como la Inspección General de Servicios del Ministerio de Economía y Hacienda, con rango de Dirección General y amplias funciones de control de gestión reconocidas en una Ley particular de 1941 .

En este mismo contexto, y si bien con dependencia funcional de la propia IGAE, las Intervenciones Generales de Defensa y de la Seguridad Social presentan también unas notas sustanciales que permiten aproximarlas más a un órgano de control de gestión que a un órgano externo $u$ horizontal.

De todos modos, la apertura clara del proceso de configuración de los órganos de control de gestión departamentales se iniciará con la aparición del Ministerio de Obras Públicas y Transportes (hoy Ministerio de Obras Públicas, Transportes y Medio Ambiente) y la creación de la Dirección General de Sistemas de Información y Control de Gestión y Procedimientos (DIGESICOGP) (Real Decreto 576/1991, de 21 de abril, con el nombre de Dirección General de Auditoría y Control de Gestión y Procedimientos; el Real Decreto 1125/1991, de 22 de julio, le da la denominación y estructura actuales).

Este proceso ha sido continuado por el Ministerio de Justicia e Interior que desde su creación incluye una Dirección General de Planificación y Control de Gestión. (Real Decreto 1334/1994, de 20 de junio). 


\section{Características del control de gestión}

Desde su definición general como función por la cual la dirección se asegura que los recursos son obtenidos y empleados eficaz y eficientemente para el logro de los objetivos de la organización, en sus acepciones más clásicas el control de gestión se ha decantado por la vía de su diferenciación del control operativo o de tareas.

El control operativo pretendería que las diferentes operaciones específicas se desarrollen conforme a los planes, eficaz y efectivamente.

Frente a ello, el control de gestión contemplaría toda la empresa (el conjunto de la organización) y le corresponde efectuar la síntesis necesaria para ofrecer esa visión global, para lo cual se centrará prioritariamente en los resultados y dentro de ellos en los denominados "factores clave de gestión", por lo que presenta un cierto carácter selectivo y parcial.

El control de gestión así entendido se configura esencialmente como un sistema de información, compuesto por los informes de resultados (informes de control) de las diferentes áreas de responsabilidad, recogiendo las desviaciones y las causas de las mismas.

Por lo general, este sistema de información se construye en base a la obtención de datos de gestión suministrados por las unidades responsables. Las últimas aportaciones de control de gestión superan ya claramente la asociación entre éste y el puro seguimiento de magnitudes contables, introduciendo con el mismo o superior nivel de importancia otras más relacionadas con los procesos y las operaciones.

En la configuración que en el apartado anterior se apuntó, el control de gestión departamental no desconoce sino que engloba toda esta construcción clásica del control de gestión, pero recoge además todo el conjunto de actuaciones específicas de revisión de procedimientos, operaciones significativas y estructuras orgánicas, en cuanto se trata de mecanismos de control dirigidos a las dos finalidades básicas de:

- Detectar e informar de desviaciones sobre las pautas marcadas por la dirección.

- Promover la mejora de la organización y su procesos.

- De acuerdo con todo ello, a nuestros efectos, podemos definir el control de gestión, como "aquél que se articula en el seno de una organización con la finalidad básica de suministrar a los órganos de dirección superior información, periódica o específica, sobre la evolución de la actividad y resultados de la estructura operativa y del cumplimiento por ésta de las diferentes reglas, internas o externas, de comportamiento, contables o de buena gestión que resulten aplicables".
- Como es normal, una definición intenta agrupar una serie de elementos que quizás convenga sistematizar, para, al mismo tiempo, situar el concepto de control de gestión dentro de la figura, más amplia y compleja, de control:

1. Control institucional: esto es, desarrollado por una determinada unidad significativa de la organización cuya función "profesional" va a ser precisamente ésa: ejercer el control de gestión.

Evidentemente la ubicación, status y composición orgánica del órgano de control serán cuestiones de no poca importancia para el ejercicio de la función, pero baste ahora señalar que debe existir la mayor proximidad entre el órgano de control y los niveles de dirección destinatarios de sus resultados.

2. Control interno: el control de gestión se articula en el seno de una organización; por lo general, por decisión de la propia organización (sin imposición externa) y ello porque no es sólo control de gestión sino control "de la dirección" de la gestión; al servicio de la dirección (de la responsabilidad máxima de encauzamiento de la gestión) y "para la gestión" en cuanto su finalidad esencial es conducir la gestión de acuerdo con los criterios de "mejor gestión" que imperen en la cúspide organizativa.

3. Suministro de información y resultados: conecta claramente con la idea de control interno antes expuesta pero, al mismo tiempo, entraña un valor en sí mismo que se relaciona además con uno de los elementos claves de la noción misma de "control", como actividad "instrumental", al servicio de otros sin ninguna nota de "autojustificación" (controlar por controlar). El control sólo servirá en cuanto sea capaz de suministrar información y/o valoraciones (no siempre tienen que ir ambos aspectos unidos); en el caso del control de gestión, a la dirección de la propia organización para:

- Ofrecer un conocimiento de la marcha de los asuntos (algo que no por elemental resulta menos importante como fuente de adopción de decisiones).

- Comunicar las desviaciones y las causas de éstas, que impliquen la necesidad de adoptar medidas correctoras de cara a los órganos operativos.

- Realimentar el proceso de adopción de decisiones, posibilitando su variación en función de la realidad puesta de relieve.

\section{Objeto del control:}

El control de gestión no encuentra limitado el contenido de la información a suministrar, por lo que en este sentido se acomoda a los diferentes tipos de control por su objeto:

- De regularidad: tanto de cumplimiento normativo como financiero o contable. 
- Operativo: de economía, eficiencia y eficacia.

- Funcional y técnico.

No puede negarse que el conocimiento sobre la adecuación de la gestión al diferente conjunto de reglas o pautas de funcionamiento que se encuadran bajo los conceptos indicados interesa, seguramente de forma primordial, a los propios responsables de la gestión.

5. Metodologia del control: nuevamente podemos afirmar que no es el método de trabajo, de análisis y de extracción de conclusiones el que caracteriza al control de gestión frente a las otras modalidades de control.

De una parte, el control de gestión puede desarrollarse por la vía del control periódico o de seguimiento de indicadores de actividad, teniendo en cuenta que la evolución y comparación temporal y funcional de los mismos permitirá a los órganos de dirección un adecuado conocimiento de la marcha del negocio.

Conviene en este punto efectuar una referencia al "control por indicadores": no se trata propiamente de efectuar una contabilidad; la información a suministrar no reunirá propiamente la característica de información contable, ni por su técnica de elaboración (en muchas ocasiones estimativas o de proyección), ni por su propio contenido (los indicadores en muchas ocasiones no consideran aspectos puramente financieros); de todos modos, no puede negarse la utilidad que la información contable representa (como un dato más) para el control de gestión, por ello, en muchas ocasiones la proximidad organizativa entre ambas funciones (Contabilidad/control) será uno de los temas a decidir en el establecimiento del control (a veces nunca decidido del todo), si bien cualquier solución debe pasar por respetar la sustantividad de ambas tareas.

Pero junto a ello, el control de gestión, de cara a su mayor operatividad, ha de comprender además la posibilidad misma de efectuar actuaciones in situ, presenciales y de examen, por vía de la técnica de "auditoria", fundamentalmente.

Estas actuaciones "auditoras" deberán realizarse para atender al interés de la organización tanto en el análisis exhaustivo de operaciones, procesos o períodos determinados, como por mantener la presencia misma del control en la organización, así como para asegurar y verificar cíclicamente la fiabilidad de los datos base para la elaboración de los indicadores de gestión, en cuanto su suministro corresponda a los propios órganos de gestión directa.

6. Relaciones con otros órganos de control: podemos considerar como una última característica del control de gestión la de que no puede desconocer la existencia de otras instancias de control, por lo general externas, que igualmente afectan al mismo objeto controlado.
Además, puede decirse que si algún control tiene un carácter "voluntario" es el control de gestión, de forma que, al menos en principio, el control externo tiene una nota de "imposición" que obliga a ser considerada necesariamente.

En este sentido, el control de gestión deberá orientarse de una forma instrumental respecto del control externo, en cuanto ello además redundará en beneficio de la propia organización; es mayor pues el "compromiso" del control de gestión en los esfuerzos por evitar todas las posibles distorsiones que el control en general puede plantear: evitar duplicidades; ofrecer actuaciones fiables en las que pueda apoyarse el control externo; servir de enlace con estos órganos; insistir y trasladar las conclusiones, promoviendo las medidas correctoras que resulten adecuadas 0 revisando las alegaciones de la propia gestión para asegurar su efectividad desde el lenguaje "común" de los órganos de control, serán tareas que ha de contener todo plan de acción de un órgano de control de gestión.

\section{El MOPTMA \\ y su estructura funcional}

Antes nos referimos al establecimiento de los primeros órganos de control departamentales en dos Ministerios como el de Obras Públicas, Transportes y Medio Ambiente (MOPTMA) y el de Justicia e Interior.

Una primera conclusión es que la aparición de estos órganos se encuentra unida a los Departamentos de estructura más amplia y compleja. En este sentido es preciso señalar que ningún órgano de control interno justifica su existencia por sí mismo. El control es una actividad conceptualmente relacionada con la estructura y dimensión de la organización controlada. Es preciso huir de formulaciones homogéneas, puesto que los distintos Departamentos de la Administración presentan sensibles diferencias en cuanto a sus funciones y su estructura y, congruentemente, deben ser tratadas de forma diferenciada situaciones distintas.

La variedad de objetivos a que debe atender el MOPTMA, con un elevado volumen de inversión a gestionar, se traduce en una compleja organización, tanto a nivel interno como en su proyección en Organismos Autónomos, Entes Públicos y Sociedades Estatales adscritos o tutelados, para la que se precisan medios humanos y materiales suficientes y adecuados.

Desde el punto de vista estructural, su esquema organizativo recoge prácticamente todas las variantes del marco común, al que nos referimos, reflejando además el "particularismo" de numerosas regulaciones personalizadas (Estatutos): 


\begin{tabular}{|c|c|}
\hline MINISTERIO & $\begin{array}{l}\text { - SECRETARIA DE ESTADO DE POLÍTICA } \\
\text { TERRITORIAL Y OBRAS PÚBUCAS } \\
\text { - SECRETARIA DE ESTADO DE MEDIO AM- } \\
\text { BIENTE Y VIVIENDA } \\
\text { - SUBSECRETARIA } \\
\text { - SECRETARIA GENERAL DE COMUNICA- } \\
\text { CIONES } \\
\text { - SECRETARÍA GENERAL DE SERVICIOS DE } \\
\text { TRANSPORTES }\end{array}$ \\
\hline $\begin{array}{l}\text { ORGANISMO AUTÓNOMO } \\
\text { COMERCIAL CON ESTATUTO } \\
\text { ESPECLAL }\end{array}$ & $\begin{array}{l}\text { ORGANISMO AUTÓNOMO CORREOS } \\
\text { Y TELÉGRAFOS }\end{array}$ \\
\hline $\begin{array}{l}\text { ORGANISMOS AUTÓNOMOS } \\
\text { COMERCIALES }\end{array}$ & $\begin{array}{l}\text { - NUEVE CONFEDERACIONES HIDROGRÁ- } \\
\text { FICAS } \\
\text { - MANCOMUNIDAD C. TAIBLIA } \\
\text { - CEDEX } \\
\text { - CENTRO ESPAÑOL DE METROLOGIA } \\
\text { - CENTRO NACIONAL DE INFORMACIÓN } \\
\text { GEOGRÄFICA } \\
\text { - PARQUE DE MAQUINARIA }\end{array}$ \\
\hline ENTES PÚBLICOS (6.1.b) & $\begin{array}{l}\text { - RENFE } \\
\text { - ENATCAR } \\
\text { - SEPES }\end{array}$ \\
\hline ENTES PÚBLICOS (6.5) & $\begin{array}{l}\text { - AENA } \\
\text { - ENTE PÚBLICO PUERTOS DEL ESTADO } \\
\text { - } 27 \text { AUTORIDADES PORTUARIAS }\end{array}$ \\
\hline $\begin{array}{l}\text { SOCIEDADES ESTATALES } \\
\text { MERCANTUES (6.1.a) }\end{array}$ & $\begin{array}{l}\text { - SENASA } \\
\text { - EMGRISA }\end{array}$ \\
\hline
\end{tabular}

En relación con las áreas de actividad propias de los diferentes Entes, el Ministerio va configurándose cada vez más como un órgano de tutela y supervisión, ejercido todo ello desde un doble nivel: la Secretaría General de adscripción directa y la Subsecretaría, como órgano horizontal, y de cierto "contraste", a través de tres unidades básicamente: Dirección General de Sistemas de Información y Control de Gestión y Procedimientos; Dirección General de Programación Económica y Presupuestaria y Secretaría General Técnica. El proceso de engranaje de estos diversos elementos no ha sido fácil ni automático y requiere un "aprendizaje con el ejercicio".

Conviene destacar no obstante que la situación actual, desde el punto de vista del MOPTMA, nos presenta ante la paradoja de la existencia de áreas de actividad prácticas de gestión sustancialmente iguales (construcción de obras públicas), pero que por su realización desde uno u otro sector (administrativo o autónomo) del Departamento se ven sometidas a regulaciones y normativas sustancialmente diferentes (obras desde la Dirección General de Carreteras o desde una Autoridad Portuaria). Los intentos de unificación de criterios generales (por ejemplo, en la valoración de los concursos) efectuados desde los órganos horizontales del Ministerio no han logrado "romper" esa sensación de diferenciación no fácilmente entendible desde las propias empresas contratistas, por ejemplo.
Desde el punto de vista del control, los Entes han generado mecanismos de "control de gestión", más en el sentido de establecimiento de líneas directrices, y suministros, periódico de información, que en la articulación de sistemas de control de operaciones o in situ, debido seguramente en parte a la continuidad lógica de los mecanismos de control "externo". (Tribunal de Cuentas, IGAE) en todo caso a posteriori.

La preocupación por el establecimiento de cauces institucionales para aumentar la transparencia en los procesos del MOPTMA no se limita a las actuaciones de la Dirección General, de forma que este órgano aparece como un elemento dentro de todo un sistema dirigido a dicha finalidad.

Se ha establecido en el Ministerio una Comisión Central de Inversiones, presidida por el Subsecretario, para la supervisión y seguimiento de las adjudicaciones de importe más relevante, con la finalidad de velar precisamente por la transparencia y regularidad de estos procesos.

También desde el primer momento de creación del Ministerio de Obras Públicas y Transportes se estableció un Centro Directivo (Dirección General de Programación Económica y Presupuestaria), específicamente dirigido al seguimiento económico y estadistico de la contratación que periódicamente suministra, tanto a los órganos de dirección como a la sociedad en general, toda una serie de información sobre la evolución en los indicadores relevantes al respecto y que, igualmente, representa un factor esencial para el seguimiento de esta actuación.

\section{Dirección General} de Sistemas de Información y Control de Gestión y Procedimientos: análisis de una experiencia

La Dirección General de Sistemas de Información y Control de Gestión y Procedimientos ha de cumplir, en el ámbito del Ministerio de Obras Públicas, Transportes y Medio Ambiente (MOPTMA) y su esfera de actuación, esencialmente la función de control interno, desarrollada desde la Subsecretaría, como módulo horizontal básico de toda la organización y a la que corresponde "la organización de las actividades de control del Departamento, realizando auditorias financieras, de gestión y de procedimientos que permitan evaluar el funcionamiento, la eficacia y el rendimiento del personal y servicios del Ministerio, sin perjuicio de las actividades que en estas materias pueda efectuar la Intervención General de la Adminis- 
tración del Estado, de acuerdo con la normativa vigente". (Art. 4.2.10, R.D. 576/1991).

La actividad de control se realiza a través de cinco Subdirecciones cuyas funciones se enumeran en el propio Real Decreto y de cuya integración resulta, pues, el conjunto de facultades propias de la Dirección. De ellas se deduce claramente que esta tarea de control, en perfecta consonancia con su naturaleza de "interno", ha de desarrollarse de forma primordial de una manera constructiva hacia la mejora del funcionamiento procedimental, la racionalización organizativa y el suministro de información adecuada y de utilidad para los diferentes niveles de dirección del Departamento, sin desconocer tampoco la de impulsar la existencia de mecanismos que aseguren la fiabilidad y regularidad de la gestión.

Las Subdirecciones que integran la Dirección General son las siguientes:

1. Subdirección General de Control Organizativo y Auditoría de Procedimientos.

2. Subdirección General de Tecnologías y Sistemas de la Información.

3. Subdirección General de Inspección de la Contratación de la Obra Pública.

4. Subdirección General de Inspección de los Servicios.

5. Subdirección General de Control de Organismos y Entes Públicos.

1. Las funciones de la Subdirección General de Control Organizativo y Auditoria de Procedimientos consisten en el análisis y revisión de la organización y de los procedimientos administrativos utilizados en las diferentes áreas de actividad del Departamento, con objeto de contrastar su eficacia y adecuación a la calidad y exigencia de los servicios públicos correspondientes, formulando las propuestas de modificaciones normativas que se estimen oportunas.

2. La Subdirección General de Tecnologias y Sistemas de la Información tiene como misión: "el análisis y diseño de los sistemas de información de gestión del Departamento necesarios para evaluar la consecución de los objetivos establecidos en los presupuestos" y "la coordinación del desarrollo informático preciso y la asistencia técnica a los Centros Directivos del Departamento en ese ámbito".

La integración en el órgano de control interno departamental de la unidad de informática, con las funciones indicadas, responde a una clara decisión estratégica que, junto con las tareas ordinarias de un Centro informático (servicio a usuarios, informática corporativa, centralización y coordinación de adquisiciones), comprende los siguientes elementos esenciales:

a) su utilización decidida en el perfeccionamiento de los sistemas de información para la dirección; b) el impulso de la Informatización de la gestión como instrumento insustituible de todo proceso de reforma y perfeccionamiento de los procesos de gestión;

c) la visión en este proceso de la informática como un elemento "instrumental" y de apoyo o complemento a las actuaciones directas de control.

3. La Subdirección General de Inspección de la Contratación de la Obra Pública tiene por cometido el análisis y control de la contratación de las obras competencia del Departamento en sus aspectos técnico, funcional y administrativo. Supone en definitiva dedicar una unidad específica de supervisión al sector de actividad fundamental del Ministerio.

En la actualidad realiza la supervisión de la gestión administrativa en materia de contratación, la inspección de obras, el informe sobre propuestas de modificación de los contratos de obras que generan un adicional económico superior al 10\% del presupuesto de adjudicación y la preceptiva recepción provisional o definitiva de las obras con presupuesto superior a 500 millones de pesetas y, de acuerdo con el Plan de Inspección previsto anualmente, la recepción de obras con presupuesto inferior al citado.

En los dos últimos años esta Subdirección ha abierto dos líneas adicionales especialmente conectadas con las características del control de gestión en este campo: el incremento de los informes globales y periódicos deducidos de sus actuaciones específicas (modificaciones, recepciones), y el seguimiento específico de la ejecución de obras a requerimiento de la Comisión de Inversiones.

4. La Subdirección General de Inspección de los Servicios, cumple las funciones de las Inspecciones Generales de todo Departamento (inspecciones de los servicios y evaluación de su gestión), salvo cuando se refieran al ámbito de la contratación de la obra pública.

5. La Subdirección General de Control de Organismos y Entes Públicos tiene por cometido el control de eficacia de los Organismos Autónomos y Entes Públicos adscritos al Departamento y que plantea la necesidad de disponer de información segmentada de ingresos, costes e inversiones asociados a los servicios o líneas sobre los que despliega su actividad cada Organismo o Ente.

Como guía y marco operativo anual, la Dirección General ha establecido en los dos últimos años un Plan de actuaciones que recoge para cada Subdirección los distintos trabajos concretos a desarrollar, agrupados en lineas de actividad significativas que se resumen en el esquema de la página siguiente.

Esquemáticamente, las directrices esenciales, de carácter general, que han marcado la actuación de la Dirección General de Sistemas de Información y Control de Gestión y Procedimientos durante 1994 y se mantienen durante 1995 serán: 
1. Profundizar en la consolidación del órgano general de control interno, partiendo de la lógica organizativa que justifica su funcionamiento en una organización con la complejidad cuantitativa y cualitativa del MOPTMA.

2. Intensificar la coordinación y vertebración entre sus Subdirecciones.

3. Potenciar las actuaciones de incidencia directa en la gestión inmediata, fundamentalmente de revisión de procedimientos operativos, con las propuestas de modificaciones oportunas.

4. ${ }^{a} \quad$ Apertura en la Subdirección General de Tecnologías y Sistemas de la Información de una línea directamente orientada a la tarea de racionalización informática de la gestión procedimental, en coordinación y apoyo del resto de las Subdirecciones.

5. Consolidar los primeros informes periódicos del área de control de gestión, respecto de Entes públicos.

6. Aumentar los niveles de información a los órganos de dirección, de los resultados de las actuaciones de control e inspección.

Todo ello dentro de un proceso en el que, en todo caso, han de solventarse una gran cantidad de dificultades y problemas prácticos que ponen de relieve que la configuración de un órgano de las características del que nos ocupa y su efectividad deben plantearse como un esfuerzo continuado en el tiempo en el que el período de cuatro años transcurridos representa tan sólo el inicio.

La fase de arranque del órgano puso de relieve además una clara descompensación de partida entre las Subdirecciones que de algún modo ya tenían antecedentes directos (Inspección de Servicios, Inspección de Contratación de la Obra Pública y Sistemas de Información) y las de nueva creación, (Control Organizativo y Auditoría de Procedimientos y Control de Organismos y Entes Públicos) que prácticamente no han dispuesto de una mínima estructura operativa hasta transcurridos tres años (cuando además se dedicaban a las funciones más "innovadoras").

El papel de un órgano de control de gestión departamental se encuentra determinado como resultante de la pluralidad de órganos de control que ejercen su actividad respecto de la misma zona considerada.

Estos órganos son tanto externos al Departamento, como más internos (dependientes de los propios agentes públicos que se sitúan en la órbita del Ministerio).

Por otro lado, la Dirección General de Sistemas de Información y Control de Gestión y Procedimientos depende de la Subsecretaría, órgano horizontal básico del Ministerio y precisamente la estructura modular de éste determina el que existan además unas importantes facultades de tutela y supervisión sobre las diferentes

\section{SUBDIRECCIONES}

INEAS DE AGTIVIDAD

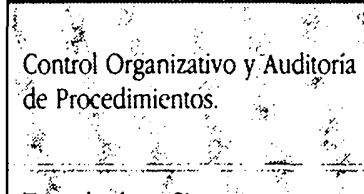

. Tecnologias y Sistemas de lá Información.

* con

organizativo.

- Auditoría de procedimientos.

- Centralización informes de control externo respecto del Ministerio.

r

- Racionalización de los procesos de gestión y mejora de los sistemas informáticos.

- Coordinación.

- Soporte y asistencia iécnica?

- Gestión de los presupuestos de inversiones y del mantenimiento informático a nivel ministerial.

- Comunicaciones.

- Formación.

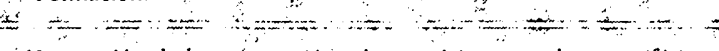

- Inspección de la contratación a los servicios centrales y periféricos.

- Inspecciones operativas a procedimientos relacionados con la contratación de obras, consultorias, servicios y suministros.

- Recepción selectiva de las obras con presupuesto inferior a 500 millones de pesetas, (O.M. 18/12/92).

- Actuaciones preceptivas sobre Recepción de obrás y Proyectos Modificados( O.M. 18/12/92 y R.D. 2534/80).

- Seguimiento espećfico de los expedientes de contratación que se ordenen a lo largo del ejercicio.

- Información sobre resultados de la actividad inspectora.

- Informaciones reservadas e inspecciones extraordinarias.

Inspección de los Servicios

- Inspécciones ordinarias de cirácter general.

- Inspecciones ordinarias operativas de servicios y centros

- Relaciones con la inspección General de Servicios de la Administración Pública.

- Inspecciones extraordinarias e informaciones reservadas.

Control de Organismos y Entes Públicos.
- Controles de gestión periódicos.- Se efectúa sobre aquellos Organismos y Entes para los que ya hay tipificados modelos de control sobre aspecros globales o parciales de su actividad y definidos sus correspondientes requerimientos de información. (RENFE; Confederaciones Hidrográficas).

- Análisis y seguimiento de informes de órganos de control externos; respecto de Organismos y Entes.

- Nuevas acruaciones de control. Ampliación del control, tanto desde el punto de vista subjetivo (nuevos Organismos y Entes) como desde el punto de vista de su ámbito material. 
áreas de actividad por parte de los órganos superiores en cada sector (Secretarías de Estado de Política Territorial y Obras Públicas y de Medio Ambiente y Vivienda; Secretarías Generales de Comunicaciones y para los Servicios del Transporte).

Así, la Dirección General de Sistemas de Información y Control de Gestión y Procedimientos ocupa un cierto carácter central entre los órganos externos de control y los sectoriales y su papel debe ser distinto en uno u otro caso (ver cuadro adjunto):

1. Por lo que se refiere a los órganos externos, el control departamental debe servir de enlace entre la estructura del Ministerio y aquellos. Puede representar un importante papel de ordenación de actividades de control y, sobre todo, de impulso interno de las medidas correctivas y de ges- tión que puedan deberse a los informes de control externos.

Ahora bien, esta función debe ejercerse sin que ello implique interferencias en el nivel de interlocución que en todo caso debe corresponder al órgano destinatario directo de la actuación de control.

2. En relación con los órganos de control de gestión de los propios Organismos o Entes, debería corresponder a la Dirección General un conjunto de funciones de impulso de su actividad, y conocimiento de sus resultados y actuaciones.

Por otro lado, la existencia de estos órganos no puede desconocerse a la hora de efectuar actuaciones de control directo (que en principio deben apoyarse en los mismos).

\begin{tabular}{|c|c|c|c|c|}
\hline & GENERAL & DEPARTAMENTAL & ESPECLAL & \\
\hline PARLAMENTO & 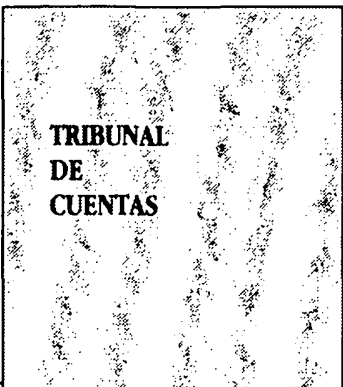 & 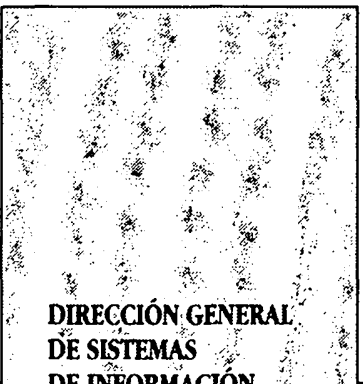 & 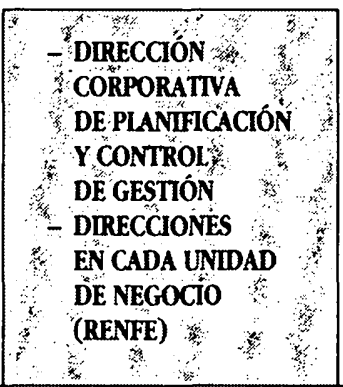 & \\
\hline $\begin{array}{l}\text { MINISTERIO } \\
\text { DE ECONOMIA }\end{array}$ & 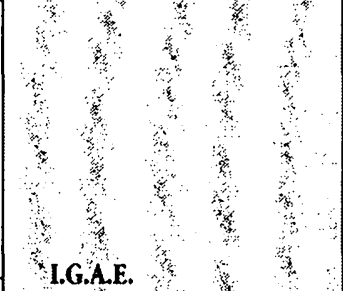 & 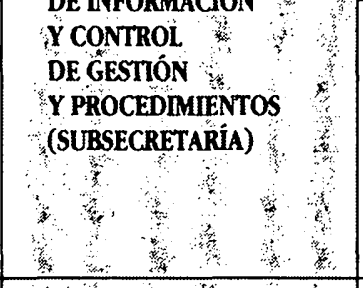 & 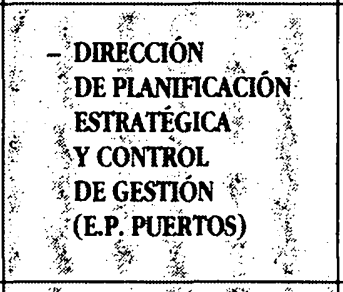 & $\begin{array}{l}\text { SECRETARIA GENERAL } \\
\text { PARA LOS SERVICIOS } \\
\text { DE TRANSPORTES }\end{array}$ \\
\hline Y HACIENDA & 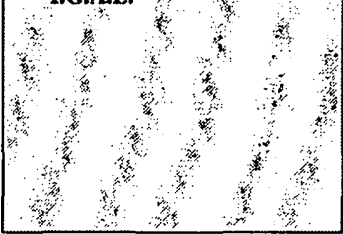 & 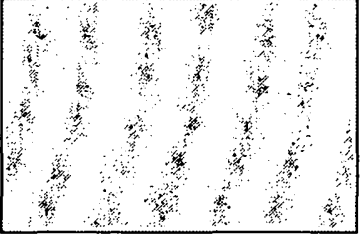 & 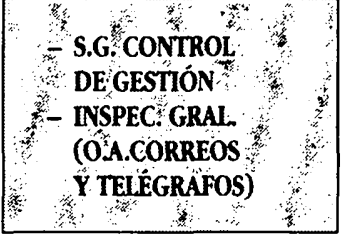 & $\begin{array}{l}\text { SECRETARIA GENERAL } \\
\text { DE COMUNICACIONES }\end{array}$ \\
\hline
\end{tabular}

\section{Reflexión final}

La aparición de los órganos de control de gestión departamentales, abre pues un campo diferente al tradicional, en el que la coexistencia entre órganos de control no se limita ya a los dos clásicos generales (TC/IGAE), sino que se amplía a una nueva figura cuya existencia no parece que deba desconocerse en el marco de ejercicio y distribución de la función genérica de control.

Uno de los aspectos a considerar para el futuro habrá de ser precisamente el de la pluralidad de controles, teniendo en cuenta además que, junto a la supresión de controles administrativos, en la práctica se produce un efecto de acumulación de un mayor número de órganos de control (con la aparición de unidades dependientes de la dirección de los propios Entes y del propio Ministerio). La coordinación en este punto resulta de gran importancia, tanto en lo que se refiere a planes de acción como a programas de trabajo para permitir un apoyo de unos órganos de control en las actuaciones de otros.

En cierto modo, la situación actual, de inicio del proceso, descansa en gran parte en relaciones de buen entendimiento y colaboración, innegables, pero que no han alcanzado los grados de for- 
malización y claridad que serían deseables para el ejercicio ordenado y regular de la función pública de control.

Estas relaciones podrían, en aras de la operatividad, formularse sobre la base de una serie de principios básicos:

$1^{\circ}$ No cuestionamiento del alcance del control a ejercer por cada nivel institucional, en función del interés efectivo del sujeto al que sirve.

2. Mantenimiento básico de las funciones y potencialidades de control de cada órgano.

3. Establecimiento formal de la regla de posible apoyo y utilización por los órganos generales de las capacidades operativas de los más concretos y cercanos a cada nivel de gestión.

4. Configuración, al menos en el nivel interno del Ejecutivo, de mecanismos de revisión de los órganos de control particulares y de "validación" de sus métodos y resultados, a fin de garantizar y posibilitar su instrumentación por el órgano general.

5. Respeto de la relación orgánica y funcional directa órgano de control particular/dirección de gestión, en cuya órbita se sitúa.

Con estas premisas, el problema puede situarse no tanto en una reconsideración de las funciones desarrolladas por cada órgano, cuanto en una redistribución de medios que tiendan a incrementar las posibilidades operativas de los órganos de control particulares, tanto por lo que respecta al efectivo cumplimiento de los requerimientos de su dirección, como para posibilitar esa actuación coordinada y "de apoyo recíproco" con el control general.

En definitiva, algo similar a lo que está ocurriendo entre los órganos de control de la Unión Europea (que habría que incluir cada vez más claramente en el catálogo de controladores de la gestión pública nacional) y determinados órganos de control nacionales, cuyas relaciones se basan en las notas de apoyo y subsidiariedad antes indicadas, todo ello dentro del respeto absoluto a los fines y razón de ser de cada control.

Como resumen, el concepto amplio de control de gestión expuesto, presenta un carácter polivalente desde distintos puntos de vista:

1. En cuanto a su objeto: alcanzando tanto al estricto control financiero o de magnitudes contables, como al control de cumplimiento o normativo y al estratégico y de economía, eficiencia y eficacia.
2. En cuanto a sus pautas de contraste o de referencia: las reglas contables o de comportamiento de gestión, el presupuesto, el plan estratégico.

3. En cuanto a su metodología: seguimiento de indicadores (tanto contables como de pura gestión) y actuaciones materiales de control. Tan sólo cabría una posible restricción, al menos en el caso concreto del sector público, en relación con el control "previo", teniendo en cuenta que el mismo es ya realizado por un órgano de control general (la IGAE), aunque al menos en vía de principios nada excluiría que la dirección de la organización pudiera arbitrar mecanismos de control preventivo para operaciones o momentos específicos.

De todos modos, el establecimiento inicial del órgano de control de gestión desde esta amplia orientación, deberá plantearse a través de un proceso, también estratégico, y dirigido hacia su progresiva institucionalización y consolidación, por encima incluso de resultados inmediatos y de cierta aparatosidad (que no resulta difícil localizar una vez detectadas debilidades estructurales); este proceso deberá articularse y construirse en función de las características existenciales del entorno en el que el control va a desarrollarse.

En este sentido y por lo que respecta al sector público, seguramente desde una perspectiva departamental, resulta más efectivo construir el control sobre actuaciones de carácter procedimental u operativo, como proceso de sedimentación del órgano, y en cuanto pueden conllevar tanto al establecimiento de una actividad periódica y programada como a generar resultados de cierta estabilidad en la organización.

Las actuaciones de control estratégico e incluso de carácter "económico" no deben desconocerse en ningún momento, ni siquiera en la fase inicial de funcionamiento, pero han de situarse en el contexto mismo de las posibilidades que la propia organización presenta para este tipo de controles y que, por lo que respecta al sector público, precisamente denotan aún grandes insuficiencias: ausencia de planificación efectiva; falta de contabilidad analítica; prioridad de los resultados cuantitativos sobre los cualitativos; así como la estrecha interconexión entre las líneas generales de acción con los criterios políticos, cuya efectiva valoración ha de entenderse alejada de las posibilidades del control de gestión, en el sentido profesional que venimos manteniendo.

- Director General de Sistemas de Información y Control de Gestión y Procedimientos - MOPTMA 


\section{Muchos \\ de los directivos de la empresa española

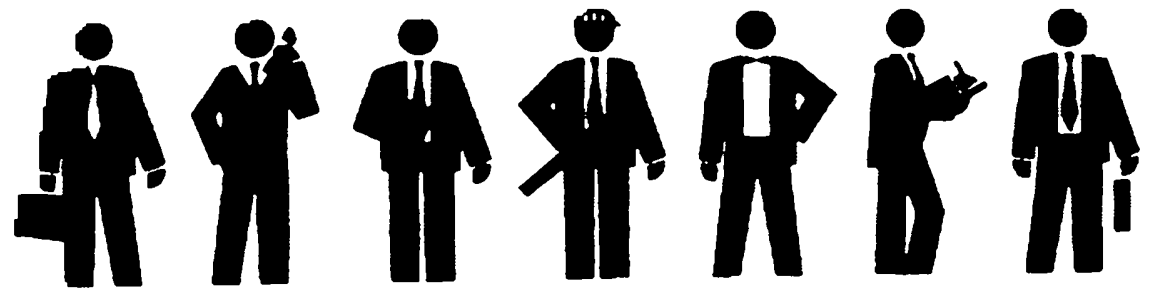 \\ se han formado en la EOI}

\section{Otros vienen para ponerse al día}

- Master en Gestión Industrial.

- Master en Gestión de Calidad.

- Master en Ingeniería y Gestión Medioambiental.

- Master en Dirección de Empresas.

- Master en Gestión de las Comunicaciones y Tecnologías de la Información.

- Master en Ingeniería Medioambiental. Gestión del Agua.

SEVIILA

Avda. n." 4. Isla de la C'artuja 41010) Sevilla

Tels.: (95) 4237567

Fax: (95) 42.37682

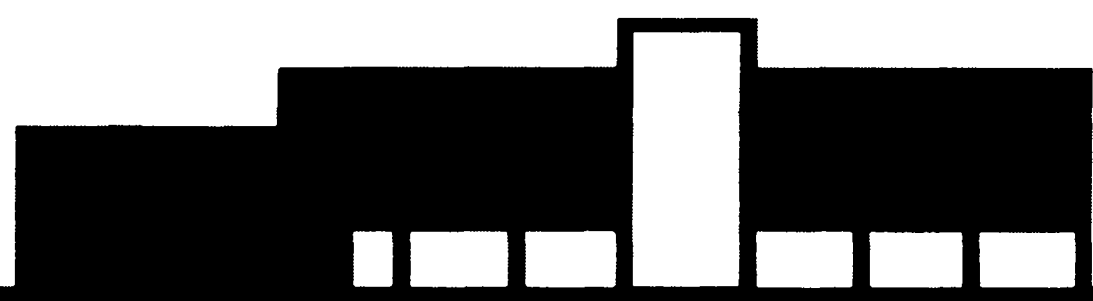

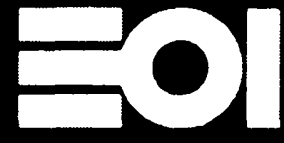

Ministerio de Industria y Energía
Escuela de Organización Industrial (iregurio del Amo. 6 (Ciudad Universitaria) 28(1)4(1) Madrid Tels.: (91) $349565(6 / 10)$ Fax: $(91) 55+2394$ 